Acta Phys. Hung. A 19/1 (2004) 000-000

HEAVY ION

PHYSICS

\title{
The 3rd Flow Component as a QGP Signal
}

\author{
L.P. Csernai ${ }^{1,2}$, A. Anderlik ${ }^{1}$, Cs. Anderlik ${ }^{1}$, V.K. Magas ${ }^{3}$, \\ E. Molnár ${ }^{1}$, A. Nyíri ${ }^{1}$, D. Röhrich ${ }^{1}$ and K. Tamosiunas ${ }^{1}$ \\ ${ }^{1}$ Section for Theoretical and Computational Physics, University of Bergen \\ N-5007 Bergen, Norway \\ 2 MTA-KFKI, Research Institute of Particle and Nuclear Physics \\ H-1525 Budapest, Hungary \\ ${ }^{3}$ Physics Department, University of valencia, Dr. Moliner 50, 46100 Burjassot, \\ Valencia, Spain \\ Received 15 May 2004
}

\begin{abstract}
Earlier fluid dynamical calculations with QGP show a softening of the directed flow while with hadronic matter this effect is absent. On the other hand, we indicated that a third flow component shows up in the reaction plane as an enhanced emission, which is orthogonal to the directed flow. This is not shadowed by the deflected projectile and target, and shows up at measurable rapidities, $y_{c} m=1-2$. To study the formation of this effect initial stages of relativistic heavy ion collisions are studied. An effective string rope model is presented for heavy ion collisions at RHIC energies. Our model takes into account baryon recoil for both target and projectile, arising from the acceleration of partons in an effective field. The typical field strength (string tension) for RHIC energies is about $5-12 \mathrm{GeV} / \mathrm{fm}$, what allows us to talk about "string ropes". The results show that QGP forms a tilted disk, such that the direction of the largest pressure gradient stays in the reaction plane, but deviates from both the beam and the usual transverse flow directions. The produced initial state can be used as an initial condition for further hydrodynamical calculations. Such initial conditions lead to the creation of third flow component. Recent $v_{1}$ measurements are promising that this effect can be used as a diagnostic tool of the QGP.
\end{abstract}

Keywords: Collective flow, Relativistic heavy ion collisions PACS: 25.75.-q, 25.75.Ld 


\section{Introduction}

In high energy heavy ion reactions we observe many thousand particles produced in the reaction, so, we have all reasons to assume that in a good part of the reaction the conditions of the local equilibrium and continuum like behavior are satisfied. The initial and final stages are, on the other hand, obviously not in statistically equilibrated states, and must be described separately, in other theoretical approaches. The different approaches, as they describe different space-time (ST) domains and the corresponding approaches can be matched to each other across ST hyper-surfaces or across some transitional layers or fronts. The choice of realistic models at each stage of the collision, as well as the correct coupling of the different stages or calculational modules are vital for a reliable reaction model.

The fluid dynamical (FD) model plays a special role among the numerous reaction models, because it can be applied only if the matter reaches local thermal equilibrium. If this happens the matter can be characterized by an Equation of State (EoS). This is what we are actually looking for in these experiments and this is why fluid dynamics is so special.

\section{The Soft Point}

The most straightforward and first observed collective flow phenomenon was the directed transverse flow. The projectile and target in the collision have overlapped, the participant region is compressed and heated up, which results in high pressure, $P$. The spectator regions, which are not compressed and heated up so much have contact with the hot central zone. The size of the contact surface, $S$, is of the order of the cross-section of the nuclei or somewhat less, depending on the impact parameter. During the collision the spectators and participants were in contact for a period of time $\tau$ and in this time the spectators acquired a directed transverse momentum component of

$$
p_{x} \approx P \times S \times \tau
$$

At a few hundred $\mathrm{MeV} /$ nucleon Beam energy the directed transverse flow momentum was almost as large as the random average transverse momentum and it was also close to the CM beam momentum.[1]

The effect was dominant, it was used to estimate the compressibility of nuclear matter, and the general expectation was that this is a good tool to find the threshold of the phase transition of the Quark Gluon Plasma. The reason is that the pressure decreases considerably versus the energy density in a first order phase transition, when the phase balance and formation of the new phase prevents the pressure from increasing in the mixed phase region. All estimates indicated that this is a strong and observable decrease in the pressure, and it has got the name the "soft point".

To some extent the expectations were fulfilled, the directed transverse flow decreased as the beam energy was increased above $1 \mathrm{GeV}$ and further. This was obvious compared to the beam momentum, but the decrease was also well observable 
compared to the average transverse momentum.

The reason is actually simple: although at high energies the pressure should increase the target and projectile are becoming more and more Lorentz contracted, and the overlap time also. So, the directed transverse momentum the non-participant matter could acquire was

$$
p_{x} \approx P \times \frac{S}{\gamma} \times \frac{\tau}{\gamma} .
$$

Thus, at high energies the directed flow could not compete with the trivial effect of reducing the flow by $\gamma^{2}$. This eliminated the interest in the directed flow, while the elliptic flow became a dominant and strong effect.

\section{The Initial State}

The elliptic flow, which is observable in the coefficient of the 2 nd harmonic, $v_{2}$, [ 2] of the flow analysis, was not effected by the increasing beam energy because it developed in the CM frame in the participant matter, due to a special symmetry in the initial state and due to the large pressure gradient in the direction of the reaction plane. The elliptic flow took over the role of the directed flow, and became an important tool in determining the basic reaction mechanism. Only models, which included a large collective pressure could reproduce the data, i.e. mainly fluid dynamical models. On the other hand, the elliptic flow effect was so strong that most fluid dynamical models could fit the data, even rather simple, one- and two- dimensional ones, so it was not a very strong diagnostic tool.

A deeper insight into fluid dynamical calculations indicated that a similar effect, arising exclusively from participant matter, should be possible to see also in the $v_{1}$ harmonic. This was first observed in 1999 [3] based on earlier FD calculations, which included a QGP EoS. The effect was named the 3rd flow component.

The effect was verified at the CERN SPS, but it was initially not detected at RHIC. Now at the beginning of this year, first STAR then two other groups have succeeded to measure the $v_{1}$ harmonic coefficient of the collective flow at RHIC also. [4, 5] This indicated to us that one needs extended theoretical studies, to map the sensitivity of this measurable.

As all fluid dynamical effects it depends on the initial state and on the EoS. It is of special interest how this effect depends on the initial state because this may shed light on the mechanisms of the formation of QGP in heavy ion reactions.

The initial state models in our recent works are based on a collective (or coherent) Yang-Mills model, a versions of flux-tube models. This approach [ 6] was implemented in a Fire-Streak geometry streak by streak and it was upgraded to satisfy energy, momentum and baryon charge conservations exactly at given finite energies [ 7, 8]. The effective string-tension was different for each streak, stretching in the beam direction, so that central streaks with more color (and baryon) charges at their two ends, had bigger string-tension and expanded less, than peripheral 
streaks. The expansion of the streaks was assumed to last until the expansion has stopped. Yo-yo motion, as known from the Lund-model was not assumed.

Our calculations show that a tilted initial state is formed, which leads to the creation of the third flow component [ [3], peaking at rapidities $|y| \approx \pm 0.75$ [ 9 ]. Recent, STAR $v_{1}$ data [4] indicate that our assumption that the string expansion lasts until full stopping of each streak, may also be too simple and local equilibration may be achieved earlier, i.e. before the full uniform stopping of a streak. We did not explicitly calculate dissipative processes, some friction within and among the expanding streaks is certainly present and experiments seem to indicate that this friction is stronger.

The expanding strings are shown in Fig. 1

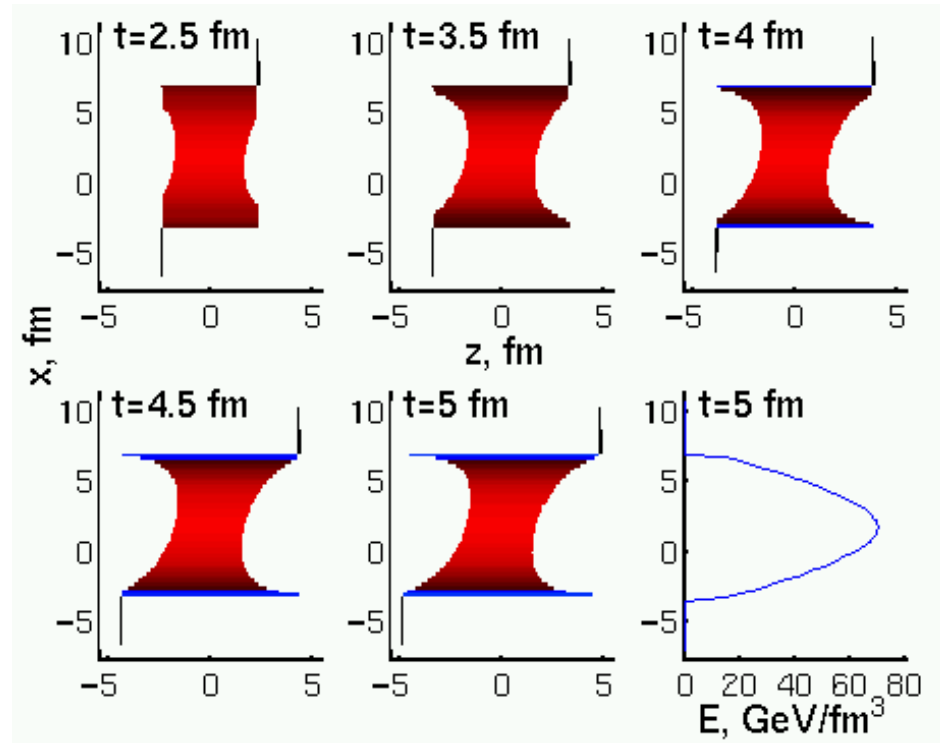

Fig. 1. $\mathrm{Au}+\mathrm{Au}$ collision at $E_{c m}=65+65 \mathrm{GeV} / \mathrm{nucl}, b=0.25 \times 2 R_{A u}$, and the parameter determining the string tension $A=0.08, E=T_{00}$ is presented in the reaction plane as a function of $x$ and $z$ for different times in the laboratory frame. The final shape of the QGP volume is a tilted disk $\approx 45^{\circ}$, and the direction of the fastest expansion will deviate from both the beam axis and the usual transverse flow direction, and will generate the third flow component. Note that the streaks are moving in the CM frame. From $[8]$

With time the streaks expand and move to the right and left on the top and bottom respectively. This motion will be reflected in the subsequent FD motion also so the tilted transverse expansion will be observed at large polar angles, i.e. relatively low rapidities. The position of the 3 rd component flow peaks depends on 
the (i) impact parameter, (ii) the effective time of the left/right longitudinal motion, (iii) the string tension determining the lengths of the streaks, (iv) the thickness of the configuration which determines the initial pressure gradient. The string tension varies as a function of the distance of a given streak from the central beam axis, because of the amount of the matter at the ends of the streaks. This determined the amount of color charge, and so the string tension is

$$
\sigma=A\left(\frac{E_{c m}}{m}\right) \frac{\sqrt{N_{1} N_{2}}}{\delta x \delta y}
$$

given in terms of the Baryon charges at the two ends of the streak, $N_{1}$ and $N_{2}$, and the cross section of the string, $\delta x \delta y$.

Although, there are several effects determining the angle of the 3rd flow component, some of these can be traced down by other measurements also: the impact parameter by the multiplicity, the effective expansion time and size by two particle correlation measurements, etc. Thus, there is a reasonable possibility that the $v_{1}$ measurements will lead to a more detailed insight to the details of the QGP formation in heavy ion reactions.

The subsequent fluid dynamical calculations show the development of the 3rd flow component from these type of initial states. These FD model calculations have to be supplemented with a Freeze Out model. There is a very essential development in this field also, and we can have different levels of approach from simplified freeze out descriptions across an FO hypersurface using an improved Cooper-Fry description, or a full kinetic description, originating from a modified Boltzmann Transport Equation approach. This final step is complicated further by the fact that the data indicate a rapid and simultaneous hadronization and freeze out.

Nevertheless, we expect that the $v_{1}$ flow data will be sufficiently detailed and accurate soon, and the 3rd flow component will be an effect which is sufficiently strong that the complex final effects at the freeze out will allow a successful analysis of the matter properties via these flow measurements.

\section{Acknowledgments}

One of the authors, L.P. Cs., thanks the Alexander von Humboldt Foundation for extended support in continuation of his earlier Research Award. The authors thank the hospitality of the Frankfurt Institute of Advanced Studies and the Institute for Theoretical Physics of the University of Frankfurt, the Gesellschaft für Schwerionenforschung, and the University of Giessen where parts of this work were done.

\section{References}

1. L. P. Csernai, Introduction To Relativistic Heavy Ion Collisions, Wiley (1994)

2. A. M. Poskanzer and S. A. Voloshin, Phys. Rev. C 58 (1998) 1671.

3. L. P. Csernai and D. Röhrich, Phys. Lett. B 458 (1999) 454. 
4. J. Adams et al., (STAR Coll.), Phys. Rev. Lett. 92 (2004) 062301.

5. M. D. Oldenburg, Poster presented at QM'04, nucl-ex/0403007 v2.

6. M. Gyulassy, L.P. Csernai, Nucl. Phys. A 460 (1986) 723.

7. V.K. Magas, L.P. Csernai, D.D. Strottman, Phys. Rev. C64 (2001), 014901. (hep-ph/0010307)

8. V.K. Magas, L.P. Csernai, D.D. Strottman, Nucl. Phys. A 712 (2002) 167-204, (hep-ph/0202085)

9. V.K. Magas, L.P. Csernai, D.D. Strottman, Proceedings of the International Conference "New Trend in High-Energy Physics" (Crimea 2001), Yalta, Crimea, Ukraine, September 22-29, 2001, edited by P.N. Bogolyubov and L.L. Jenkovszky (Bogolyubov Institute for Theoretical Physics, Kiev, 2001), pp. 193-200; and hep-ph/0110347 